\title{
Emprendimiento, creatividad e innovación en la gestión empresarial del Ecuador
}

\section{Entrepreneurship, creativity and innovation in business management in Ecuador}

\author{
María Fernanda Salazar Bonilla \\ msalazar@edu.ec \\ Pontifica Universidad Católica del Ecuador, Ambato \\ Ecuador \\ https://orcid.org/0000-0002-5543-7643 \\ Helder Marcell Barrera Erreyes \\ hm.barrera@uta.edu.ec \\ Universidad Técnica de Ambato/ Pontificia Universidad Católica del Ecuador, \\ Ambato \\ Ecuador \\ https://orcid.org/0000-0002-2157-3778 \\ Luis Gabriel Nuñez Torres \\ gnunez@uti.edu.ec \\ Universidad Tecnológica Indoamérica/ Pontificia Universidad Católica del Ecuador, \\ Ambato \\ Ecuador \\ https://orcid.org/0000-0002-1891-4455 \\ Fernando Alfredo Flor Tapia \\ fflor@pucesa.edu.ec \\ Pontificia Universidad Católica del Ecuador, Ambato \\ Ecuador \\ https://orcid.org/0000-0001-5766-3444
}

Recibido: 15 de junio de 2019

Aprobado: 1 de agosto de 2019

\section{RESUMEN}


Este artículo se propuso como objetivo evaluar el emprendimiento, creatividad e innovación en la gestion empresarial del ecuador. Se auxilió de una metodologia documental - descriptiva, apoyada en documentos para el análisis hermenéutico de las teorías que sustentan la variable en un estudio y orientada a redactar informes relacionados con el estado real de las personas, objeto, situaciones 0 fenómenos tal cual se presentan en el momento de su recolección, describe lo que mide sin analizar inferencias ni verificar hipótesis. La población la constituyeron 43 empresas que hacen vida comercial en distintos cantones del Ecuador. La recaudación de la información se hizo con un instrumento tipo cuestionario, con características de una lista de cotejo. De acuerdo con su intención, la misma asumió una postura epistemológica basada en el idealismo-deductivo. En sus conclusiones, se indicó que la gestion empresarial del ecuador, está condicionada a un proceso poco ordenado y uniforme.

Descriptores: Emprendimiento; Creatividad; Innovación; Gestion empresarial; Empresarios.

\begin{abstract}
This article aimed to evaluate entrepreneurship, creativity and innovation in the business management of the equator. A documentary-descriptive methodology was helped, supported by documents for the hermeneutic analysis of theories that underpin the variable in a study and aimed at writing reports related to the actual state of people, object, situations or phenomena as presented at the time of collection, describes what measures without analyzing inferences or verifying hypotheses. The population consisted of 43 companies that make commercial life in different cantons of Ecuador. The collection of the information was through a questionnaire-type instrument, with characteristics of a checklist. According to her intention, it assumed an epistemological stance based on idealism-deductive. In its conclusions, it was stated that the business management of the equator is conditioned on an unordered and uniform process.
\end{abstract}

Descriptores: Entrepreneurship; Creativity; Innovation; Business management; Entrepreneurs.

\title{
INTRODUCCIÓN
}

La creatividad, la innovación y el emprendimiento, se han convertido hoy en día, como parte fundamental del capital humano. La importancia de estos elementos 
deriva del desarrollo de nuevos procesos, productos y servicios, que asegurarían la supervivencia y el éxito de las empresas. Sin embargo, existen algunos componentes, que al no ser considerados, entre estos la innovación, la creatividad y el emprendimiento, para con las empresas generan deficientas en la gestion de las mismas. Por ello, queda pensar tal como lo exponen Catmull y col (2008), que el origen de estos componentes no sólo se encuentra en el capital humano de la empresa, sino fuera de ella, de manera que, aunque el personal forme parte del proceso de creación, las iniciativas vengan de individuos externos a la empresa ya sean los propios consumidores o el público en general a través de encuestas, buzones de sugerencias o incluso plataformas de co-creacción como pueden ser las wikis.

Ante ello, hay que ser sensatos en relación a los elementos de creatividad, innovación y el emprendimiento que afectan a las empresas, entendiendo que no solo son muy numerosos y variados si no que muchos de ellos no son controlables por los responsables de su gestion. Cabe, destacar que cada empresa es un mundo en sí mismo, y ha de contar con otros muchos recursos imprescindibles para el desarrollo de su actividad que también deberá gestionar y organizar con dos objetivos fundamentales (Bartlett y col, 1993). Con base en lo expuesto, este artículo se permitió evaluar la gestion empresarial del ecuador en términos de su emprendimiento, creatividad e innovación.

\section{DESARROLLO}

Bessant y Tidd, (2007), indican que la relación entre creatividad, innovación y emprendimiento se puede entender de la siguiente manera: la creatividad es una capacidad que unida al comportamiento y a la actitud a la hora de aprovechar oportunidades trae consigo el emprendimiento y da lugar a grandes innovaciones muy beneficiosas para la empresa y los individuos. 


\section{La creatividad}

Runco y col (2012), expresan a la creatividad como la capacidad de generar y hacer realidad nuevas ideas, todo considerando necesario tanto la originalidad como la eficacia. Esta última, entendida como la capacidad de llevar a cabo las ideas generadas, diferenciándola de la imaginación. Por su parte, Catmull y col (2008), indican que en la práctica el proceso creativo conlleva la unión de muchas ideas y la colaboración de muchas personas con diferentes conocimientos que tienen una grandísima influencia en todas las fases que hacen que la idea inicial tome forma dando lugar a innovaciones.

\section{La innovación}

Según la Organización para la Cooperación y el Desarrollo Económicos (OCDE): «La innovación es un proceso iterativo activado por la percepción de una oportunidad proporcionada por un nuevo mercado y/o nuevo servicio y/o avance tecnológico que se puede entregar a través de actividades de definición, diseño, producción, marketing y éxito comercial del invento». La innovación es un proceso multifacético que se ha definido en varias ocasiones como la búsqueda de nuevas formas de hacer las cosas.

\section{La innovación como proceso}

Entendiendo la innovación como un cambio que incluye la creación y comercialización de conocimiento (Tidd, Bessant, y Pavitt, 2001), sus asociados infieren que aunque la creatividad juegue un papel fundamental, la necesidad de implementar y comercializar las ideas hace de la innovación un proceso que consta de diferentes fases, tales como; fomentar y registro de ideas; selección de ideas, evaluación, gestión del portfolio; Desarrollo de producto y de gestión del proyecto y una última de inventario y/o ventas. (Oke, Munshi, y Walumbwa, 2009). 


\section{La innovación como actividad}

También puede entenderse como una actividad organizacional basada en distintos grados de novedad. De esta forma podemos encontrar innovaciones radicales e innovaciones incrementales o, como decía James Idea Generation Screening, en cuanto a la producción de las ideas, refieren a: fomentar la generación, inventario / ventas de ideas, registro, selección de ideas, evaluación y gestión del portfolio llustración

\section{La innovación como proceso - Desarrollo de producto - Gestión del proyecto} (Oke, Munshi, y Walumbwa, 2009) Creatividad, Innovación y Emprendimiento en la empresa: Análisis y diagnóstico de los factores internos Ana Maria Montoya Flores 5 March "Exploración de nuevas posibilidades y explotación de viejas certezas". En ambos casos será un conjunto diferente de actividades, habilidades, recursos y gestión de los mismos los que harán posible que esta innovación llegue a producirse (Oke, Munshi, y Walumbwa, 2009).

- Innovaciones radicales: Implican el descubrimiento e implantación de algo totalmente nuevo. En este caso la creatividad asume un papel protagonista y, en muchos casos será necesaria una tecnología y una sofisticación que permita desarrollar y evaluar las actividades y procesos necesarios para su consecución. Entre estas actividades, que podemos clasificar en su mayoría como actividades de exploración, podemos incluir la investigación, la experimentación y la asunción y medición de riesgos.

- Innovaciones incrementales: Buscan una mejora de algo ya existente. En este caso, a diferencia de lo que ocurre en el desarrollo de innovaciones radicales, la creatividad, aunque sea necesaria en menor medida, pierde importancia frente a otro tipo de actividades que podemos denominar actividades de explotación. Entre ellas se encuentran el desarrollo de productos, el reposicionamiento de la oferta y la optimización de procesos y servicios. 


\section{Emprendimiento}

Se conoce como emprendimiento a la actitud y aptitud que toma un individuo para iniciar un nuevo proyecto a través de ideas y oportunidades. El emprendimiento es un término muy utilizado en el ámbito empresarial, en virtud de su relacionamiento con la creación de empresas, nuevos productos o innovación de los mismos. (García, 2016).

Aunque la definición más extendida de emprendimiento sigue estando relacionada con la creación de empresas, también se puede entender el emprendimiento desde una visión mucho más amplia. De hecho, Herrera, (2009), realizó un estudio en el que analizó más de noventa definiciones obtenidas de directivos y académicos. A pesar de no haber podido alcanzar una única definición válida para todos los contextos, sí encontró una serie de conceptos que se repetían si no en todas, en la inmensa mayoría de los casos, entre estos: comenzar, fundar o crear; nuevo negocio, empresa o iniciativa; innovación, nuevos productos o nuevos mercados; búsqueda de oportunidades; asunción y gestión de riesgo e incertidumbre y búsqueda de lucro y beneficio persona.

\section{Emprendimiento organizacional}

Al igual que ocurre a la hora de definir el emprendimiento a grandes rasgos, hay muchas definiciones de lo que podemos entender como emprendimiento organizacional; en general, es un término que se ha usado para describir un comportamiento emprendedor dentro de organizaciones ya establecidas, aunque también podemos encontrar definiciones más precisas.

Entre ellas, ha sido definido como un constructo multidimensional aplicado a nivel organizacional que caracteriza el comportamiento emprendedor en la empresa y que incluye al menos una de las siguientes dimensiones; asunción de riesgos, innovación y proactividad.( Ruiz y col, 2015). 
Aunque en muchas ocasiones son usados como sinónimos, emprendimiento organizacional e emprendimiento no son totalmente equivalentes: Emprendimiento implica dotar a una división de cierto grado de autoridad a la hora de tomar decisiones pero siempre en torno a la creación de beneficio económico. De alguna forma el emprendimiento funciona de la siguiente manera: un inventor dentro de una gran compañía forma su pequeña empresa dentro de la misma (subdivisión) y la gestiona aparte con cierto grado de autonomía (Ruiz y col, 2015). Así, podemos entender que el emprendimiento es un fruto de la innovación, mientras que el emprendimiento organizacional, según las definiciones comentadas en el apartado anterior, es una fuente de la misma. Aldana \& Piña (2018), señalan que es necesario evaluar las perspectivas del cliente para que la organización preste un servicio de calidad. Autores como Vicens y col (2011), señalan que el emprendimiento organizacional está basado en los siguientes conceptos:

Autonomía; Se refiere a las acciones llevadas a cabo por individuos o equipos de manera independiente con el objetivo de sacar adelante un concepto de negocio $o$ visión hasta ser completadas.

Innovación; Consiste en introducir intencionadamente la novedad a través de la experimentación y procesos creativos que buscan el desarrollo de nuevos productos y servicios así como nuevos procesos.

Proactividad; Se trata de la asunción de una perspectiva de futuro por parte del líder de un mercado que tiene la capacidad de aprovechar las oportunidades anticipándose a la demanda futura.

Competencia agresiva; Consiste en realizar un gran esfuerzo para mejorar a los otros rivales en la industria. Se caracteriza por tomar una posición de ataque o responder de manera agresiva ante los competidores con el objetivo de mejorar su posicionamiento o superar las amenazas de un mercado muy competitivo.

Asunción de riesgos; Implica tomar decisiones y actuar en un entorno de incertidumbre donde se desconocen todos los posibles resultados. En algunos 
casos puede requerir la inversión de una considerable cantidad de recursos para la consecución final del proyecto. Dicho esto, podemos concluir con que el emprendimiento organizacional se materializa desde 3 posibles perspectivas (Stopford and Baden-Fuller, 1994)

Corporate Venturing o imprendimiento. Conlleva la creación de una nueva línea de negocio dentro de una organización ya consagrada.

Corporate Renewal o renovación empresarial. Es la forma en la que las empresas buscan modificar la forma en la que opera la organización existente y su forma de competir en el mercado.

Corporate Leadership o liderazgo corporativo; La organización quiere cambiar las normas que rigen la manera en la que los diferentes agentes compiten en la industria.

\section{METODOLOGIA}

La metodología asumida en el presente artículo se centró en una investigación documental bibliográfica y descriptiva. Para Chavez (2008), lo documental es aquello que se apoya en documentos para el análisis hermenéutico de las teorías que sustentan las variables en un estudio. En cuanto a los estudios descriptivos, indican Hernández y col (2014), es la que se orienta a redactar informes relacionados con el estado real de las personas, objeto, situaciones o fenómenos tal cual se presentan en el momento de su recolección, sin realizar inferencias ni verificar hipótesis. La población la constituyeron 43 empresas que hacen vida comercial en distintos cantones del Ecuador. La recolección de la información fue a través de un instrumento tipo cuestionario, con características de una lista de cotejo. De acuerdo con su intención, la misma asumió una postura epistemológica basada en el idealismo-deductivo, para lo cual se partió del conocimiento previo de las teorías de emprendimiento, creatividad e innovación 
para confrontarla con los datos obtenidos de los informantes y presentar conclusiones hermenéuticas de carácter objetivo.

\section{RESULTADOS}

-Se pudo constatar que la creatividad, en el marco de la gestion empresarial ecuatoriana, se observa como un proceso poco ordenado y uniforme, sin permitir que se interrelacionen, cambian de orden y se crean sinergias según la idea para poder desarrollarse, que en muchos casos los lleva a auxiliarse de las imitaciones. -En la innovación como proceso, se puede indicar una gestion empresarial factible manejarse desde la tolerancia a la ambigüedad y al fracaso así como a una línea de comunicación desestructurada.

-Es de indicar, que en fases más avanzadas del proceso como es el desarrollo de prototipos, la producción y la distribución para la existencia de las empresas, en su mayoría carecen de una organización clara y estructurada que permita a los distintos departamentos y partes involucradas coordinarse para lograr comercializar a tiempo y con éxito sus productos.

-Para las innovaciones incrementales, se requiere de una gestion diferente que se inicie desde la misma organización y está fundamentada en estrategia que permitan el incremento de las innovaciones, si se toma en cuenta que debe existir un equilibrio, entre la innovación y las actividades, para lograr penetrar de forma exitosa

A sus entornos comerciales.

-Se constató poca disposición, dentro de la gestion empresarial, de aquellos ingredientes esenciales para asumir riesgos relacionados con el tiempo, capital y carrera profesional. Asimismo, pocas manifestaciones de habilidades para formular un equipo efectivo; reunir recursos de forma creativa; construir un plan 
de negocio sólido y tener una visión necesaria para reconocer las oportunidades donde otros ven caos, contradicciones y confusión.

\section{CONCLUSIONES}

Se pudo constatar que la gestion empresarial del ecuador, está condicionada a un proceso poco ordenado y uniforme, sin permitir que se interrelacionen, cambien de orden y se creen sinergias según la idea para poder desarrollarse, auxiliándose de las imitaciones. Tambien existe un manejo de la tolerancia a la ambigüedad y al fracaso y en términos de una comunicación desestructurada.

Por otra parte, se carece de una organización clara y estructurada que permita a los distintos departamentos y partes involucradas de las empresas, coordinarse para lograr comercializar a tiempo y con éxito sus productos, a partir de una gestion diferente que se inicie desde la misma organización y esta fundamentada en estrategias que permitan el incremento de las innovaciones.

Fue posible, constar poca disposición, dentro de la gestion empresarial, para considerar aquellos ingredientes esenciales que permiten asumir riesgos relacionados con el tiempo, capital y carrera profesional.

\section{REFERENCIAS CONSULTADAS}

1. Aldana, J., \& Piña, J. (2018). Calidad del servicio prestado al cliente por los instructores de gimnasios. Revista Arbitrada Interdisciplinaria Koinonía, 2(3), 172-197. Recuperado de http://fundacionkoinonia.com.ve/ojs/index.php/revistakoinonia/article/view/59 $\underline{146}$

2. Bartlett, C. A., \& Ghoshal, S. (1993). Beyond the m-form: toward a managerial theory of the firm. Strategic Management Journal, 23-45.

3. Bessant, J., \& Tidd, J. (2007). Innovation and Entrepreneurship. Chichester: John Wiley \& Sons. 
4. Chávez, N (2008).Introducción a la Investigación Educativa. 4ta edición. Editorial EDILUZ. Maracaibo, Venezuela

5. García, L. (2016). Emprendimiento digital: estudio de caso con universitarios de comunicación, UAEM UAP Huehuetoca. Revista de Ciencias. Sociales, (29), pp. 34-45

6. Hernández R, Fernández C y Baptista P (2014). Metodología de la Investigación. Chile: McGraw - Hill.

7. Herrera, H. (2009). Investigación sobre redes sociales y emprendimiento: revisión de la literatura y agenda futura. Innovar. Volumen 19, Número 33, p. 19-33, 2009. ISSN electrónico 2248-6968. ISSN impreso 0121-5051.

8. Oke, A., Munshi, N., \& Walumbwa, F. O. (2009). The Influence of Leadership on Innovation Processes and Activities. ScienceDirect, 64-72.

9. Ruiz, M., Arroyo, I., Espinoza, Sanz y Fuentes, M. (2015). lidentificación de oportunidades emprendedoras. Elsevier, 21(2015),47-5M. DOI: 10.1016/j.iedee.2014.07.0021135-2523/@2014AEDEM.

10. Runco, M. A., \& Garrett, J. J. (2012). The Standard Definition of Creativity. Creativity Research Journal, 92-96.

11. Vicens, L y $\quad$ Grullón, S. $\quad$ (2011). Innovación y emprendimiento: Un modelo basado en el desarrollo del emp rendedor. Presentado en el V Foro de Competitividad de las Américas para el Banco Interamericano de Desarrollo y el Compete Caribbean Santo Domingo, República DominicanaSabino, C. (2010). El Proceso de Investigación. Editorial Panapo. Caracas.

\section{REFERENCES CONSULTED}

1. Aldana, J., \& Piña, J. (2018). Quality of service provided to the client by gym instructors. Interdisciplinary Arbitrated Review Koinonía, 2 (3), 172-197. Recovered from http://fundacionkoinonia.com.ve/ojs/index.php/revistakoinonia/article/view/59 $\underline{146}$

2. Bartlett, C. A., \& Ghoshal, S. (1993). Beyond the m-form: toward a managerial theory of the firm. Strategic Management Journal, 23-45. 
3. Bessant, J., \& Tidd, J. (2007). Innovation and Entrepreneurship. Chichester: John Wiley \& Sons.

4. Chávez, N (2008). Introduction to Educational Research. 4th edition EDILUZ editorial. Maracaibo Venezuela

5. García, L. (2016). Digital entrepreneurship: case study with university students, UAEM UAP Huehuetoca. Science Magazine Social, (29), pp. 3445

6. Hernández R, Fernández C and Baptista P (2014). Investigation methodology. Chile: McGraw - Hill.

7. Herrera, H. (2009). Research on social networks and entrepreneurship: literature review and future agenda. Innovate. Volume 19, Number 33, p. 1933, 2009. Electronic ISSN 2248-6968. ISSN printed 0121-5051.

8. Oke, A., Munshi, N., \& Walumbwa, F. O. (2009). The Influence of Leadership on Innovation Processes and Activities. ScienceDirect, 64-72.

9. Ruiz, M., Arroyo, I., Espinoza, Sanz y Fuentes, M. (2015). Identification of entrepreneurial opportunities. Elsevier, 21 (2015), 47-5M. DOI: 10.1016 / j.iedee. 2014.07.0021135-2523 / @ 2014AEDEM.

10. Runco, M. A., \& Garrett, J. J. (2012). The Standard Definition of Creativity. Creativity Research Journal, 92-96.

11. Vicens, L y Grullón, S. (2011). Innovation and entrepreneurship: A model based on the development of the entrepreneur. Presented at the $\mathrm{V}$ Competitiveness Forum of the Americas for the Inter-American Development Bank and the Caribbean Competition Santo Domingo, Dominican Republic Sabino, C. (2010). The Research Process Panapo editorial. Caracas. 